\title{
Effect of dietary selenium intake on gut microbiota in older population in Enshi region
}

\author{
Zi-xiong Zhang ${ }^{1,2+}{ }^{\text {, Hua Xiang }}{ }^{2 \dagger}$, Guo-gen Sun ${ }^{1,2}$, Yan-hua Yang ${ }^{1,2}$, Chen Chen ${ }^{3}$ and Tuo Li $\mathrm{Li}^{1,{ }^{*}}$
}

\begin{abstract}
Background: The microbial ecosystem in the human gut varies between individuals with differences in diet. Selenium is one of most common trace elements in everyday diet, and selenium intake affects the human gut microbiota. We studied the effect of selenium intake on the gut microbiota in regions of Enshi with different distributions of selenium.
\end{abstract}

Methods: One hundred elderly subjects ( $>65$ years) were recruited from high-selenium and low-selenium areas in Enshi and blood, nail, and fecal specimens were obtained. The selenium contents in these samples were determined in triplicate by hydride generation atomic fluorescence spectrometry. DNA was extracted from fecal specimens and the microbial diversity was analyzed by 16 S RNA.

Results: The selenium contents in the blood and nails were significantly different between the high- and lowselenium areas, and the composition of the intestinal microbiota, including abundance and extent of intestinal flora, was altered. The function and metabolic pathways of the gut microbiota showed clear differences.

Conclusions: As a trace element in human diet, selenium intake is an important factor that affects the intestinal microbiota and is likely involved in many human diseases. This study provides new clues and ideas for studying the correlation between selenium and human health.

Keywords: Selenium, Gut microbiota, 16S RNA, Diet, Enshi

\section{Introduction}

Human intestines occupy a fairly large surface area ranging from 30 to 400 cubic meters, [1], and the gut harbors more than $10^{14}$ microbes [2]. Human gut microbial communities are composed of the dominant phyla Firmicutes and Bacteroidetes and very low proportions of Proteobacteria, Acinobacteria, Fusobacteria, and Verrucbacteria [3]. They consist of approximately 1150 prevalent species, most of which are shared in population, and

\footnotetext{
* Correspondence:_zhxs@163.com

${ }^{1}$ Institute of Selenium and Human Health of Hubei, Hubei Province, Enshi City, China

${ }^{2}$ Central Hospital of Enshi Autonomous Prefecture, Enshi Autonomous Prefecture, Hubei Province, Enshi City, China

Full list of author information is available at the end of the article
}

at least 160 such species are present in each individual $[4,5]$. The gut microbiota is regarded as a "superorganism" and vastly expands on the functional capabilities provided by our genomes. The gut microbiome is estimated to contain 150 times more genes than the human genome [6]. Numerous studies have revealed that intestinal microbiota affect many aspects of host physiology including nutrient absorption, vitamin production, metabolic phenotype [7], and immune system development and regulation $[7,8]$. The dysbiosis of intestinal microbiota could lead to host disease such as diabetes type 1 and type 2 [9], irritable bowel syndrome [10], colonic cancer [11], and inflammatory bowel disease [12]. 
Recent studies have indicated that the microbial ecosystem in the human gut varies between individuals based on country of origin, lifestyle, age, and health status [6]. The microbial ecosystem mainly reflects certain functional core microbiomes, wherein wide arrays of microbial genes are shared and identified [13]. Moreover, the functional core microbiomes are associated with different host phenotypes including country of origin and gender, leading to the proposal of three predominant "enterotypes": Bacteroides, Prevotella, and Ruminococcus [14]. The aging process significantly affects human gut microbiota communities because of age-related physiological changes such as digestive hormone secretion, intestinal morphology, nutrient absorption, and functionality of the host immune system [15]. Diet was hypothesized to have a more significant impact on the microbial ecosystem and plays a crucial role in shifting the intestinal microbiota. Food source could lead to changes in circular pheromones in the host by affecting symbiotic bacteria [16]. Diets with high caloric content, high fat content, or low fiber content may change alter composition of the intestinal microbiota [17].

Trace elements, which are important in the composition of a diet, can also affect the intestinal microbiota. For example, iron supplementation contributes to the re-establishment of the original gut microbiota composition [18]. Selenium is one of most common trace elements in the diet, but few studies have reported the effect of selenium on the intestinal microbiota. The effect of selenium intake on the gut microbiota require further investigation. Enshi has the only separate selenium mines in the world, which were formed during the Maokou, Late Permian period. Carbon-siliceous sediments, also known as "stone coal", contain the highest content of selenium (up to $8,500 \mathrm{mg} / \mathrm{kg}$ ) [19]. Human activities have played important roles in manifesting a special geographical environment with uneven distributions of Se. We herein studied the effect of selenium intake on the gut microbiota in areas with different distributions of selenium.

As a trace element, selenium plays a variety of important roles in organisms, of which the presence of selenoprotein in the form of selenium plays an important role. The physiological metabolic activities that selenoprotein participates in include the body's peroxide damage repair, participation in the regulation of hormone activity, and lipid component metabolism. Selenium in selenium. The existing form of protein is selenocysteine, and selenoenase is the most important type of selenoprotein. Food is the main way for the body to take in selenium. Selenium is widely found in foods such as eggs, fish, grains, meat, fruits, vegetables, nuts, etc., and it exists in the form of inorganic selenite. selenate, they are absorbed by plants from the soil and converted into organic form, in the form of selenomethionine and selenocysteine, Humans can obtain selenium by ingesting plant or animal food. Therefore, to study the effects of selenium on the human body, the soil selenium content can be used as a reference basis [20].

\section{Materials and methods instrumentation}

\begin{tabular}{ll}
\hline Instrument Name & Model \\
\hline DNA isolation kit & $\begin{array}{l}\text { PowerMax (stool/soil) DNA isolation kit (MoBio } \\
\text { Laboratories) }\end{array}$ \\
$\begin{array}{l}\text { Atomic fluorescence } \\
\text { spectrometry }\end{array}$ & $\begin{array}{l}\text { Titan instruments AFS-921 (Beijing Titan) } \\
\text { Sample collecter }\end{array}$ \\
$\begin{array}{l}\text { Beckman Coulter Hemoccult II SENSA }{ }^{\oplus} \text { cards } \\
\text { (Beckman Coulter, CA) }\end{array}$ \\
$\begin{array}{l}\text { DNA extract and } \\
\text { Spectrophotometer }\end{array}$ & $\begin{array}{l}\text { NanoDrop } 1000 \text { spectrophotometer (Thermo } \\
\text { Scientific, USA) }\end{array}$ \\
DNA sequencer & Illumina Hiseq4000 sequencer \\
\hline
\end{tabular}

\section{Ethical considerations}

This study was conducted in accordance with the guidelines of the Declaration of Helsinki, and all procedures involving human subjects were approved by the Ethics Committee of the Central Hospital of Enshi Autonomous Prefecture. All subjects were given oral and written information about the purpose and procedures of the study. Consent to participate was signed by the subjects before the study started, and the subjects were free to withdraw from the study at any time point without giving any explanation.

\section{Participants}

Subjects were recruited from high-selenium and lowselenium areas of Enshi, Hubei province, China. In total, more than 260 elderly (>65years) male and female adult volunteers were invited for screening. From this pool of volunteers, 100 subjects were randomized to participate in the study. The inclusion criteria were as follow: (1) lived in this area for more than ten years; (2) ability and willingness to understand and comply with the study procedures and sign the written informed consent; (3) no special diet customs; (4) willingness to provide blood, nail, and fecal specimens; (5) no use of antibiotic, antiviral, or antifungal drugs within the nearest month; and (6) blood selenium patiancecontent is $>120 \mu \mathrm{g} / \mathrm{L}$ in high-selenium area and $<80 \mu \mathrm{g} / \mathrm{L}$ in low-selenium area. The exclusion criteria were as follows: (1) suffered from acute gastrointestinal disease recently; (2) subjected to acute gastrointestinal surgery recently; (3) residents of abnormal nutritional status, such as terminal cancer 
patients or patients with cerebral apoplexy; and (4) did not provide blood, nail, or fecal specimens.

\section{Laboratory analyses}

The selenium contents in all samples were determined in triplicate by hydride generation atomic fluorescence spectrometry.

\section{Fecal sample collection}

Fecal samples were collected from the subjects using the two sections of Beckman Coulter Hemoccult II SENSA ${ }^{\circ}$ cards (Beckman Coulter, CA) at home. The volunteers were told to collect specimens in advance, and the specimen should come from the end of the stool while avoiding contact with urine or other possible pollutants. The collected samples were immediately placed in a prepared centrifuge tube, which were kept in ice and sent to our laboratory. The samples were placed into plastic bags and stored at $-80{ }^{\circ} \mathrm{C}$ until processed further. DNA was extracted from the $0.18-0.2 \mathrm{~g}$ fecal samples in accordance with the instructions of the QIAamp DNA Stool Mini Kit (Qiagen, USA). The DNA concentration and quality were determined by a NanoDrop 1000 spectrophotometer (Thermo Scientific, USA).

\section{Measurement of gut microbiota DNA extraction and rDNA sequencing}

DNA was extracted from all samples using the PowerMax (stool/soil) DNA isolation kit (MoBio Laboratories). All paired samples were evaluated by $16 \mathrm{~S}$ rDNA primer (amplicon) Sequencing (515F (5'-GTGC CAGCMGCCGCGGTAA-3') and 806R (5'-GGACTA CHVGGGTWTCTAAT-3')). Libraries of $16 \mathrm{~S}$ rDNA amplicons were prepared by targeting the V4 hypervariable regions of the rDNA. The amplicons were attached with adapters, and indexes were added in a subsequent nested polymerase chain reaction $\left(98{ }^{\circ} \mathrm{C}\right.$ for $30 \mathrm{~s}, 8 \times\left(98{ }^{\circ} \mathrm{C}\right.$ for $10 \mathrm{~s}, 55{ }^{\circ} \mathrm{C}$ for $20 \mathrm{~s}, 72{ }^{\circ} \mathrm{C}$ for $\left.20 \mathrm{~s}\right)$, $72{ }^{\circ} \mathrm{C}$ for $\left.5 \mathrm{~min}\right)$. Products from polymerase chain reaction were pooled and the purified with magnetic beads and sequenced on the Illumina Hiseq4000 sequencer $(2 \times 150$ bp paired end $)$.

\section{Reads processing and metagenomic clustering}

We used QIIME v1.9.0 to process reads and trim additional quality, demultiplexing [21]. Operational taxonomic unit (OTU) was picked using Vsearch v1.11.1, dereplicating, clustering, detection of chimeras [22]. Taxonomic assignment of individual datasets was performed using SILVA128. Alpha diversity was performed with qiime, including index of observed species. Beta diversity was calculated using QIIME with the matrix of (weighted and unweighted) Unifrac distance. Functional profiling of microbial communities was created with PICRUSt1.1.0 to predict the using $16 \mathrm{~S}$ rRNA sequences. Further analysis was processed with Statistical Analysis of Metagenomic Profiles (STAMP) software package v2.1.3 [23]. The beta diversity on taxonomy and functions based on Meta-Storms distances were generated by Parallel-META 3 (version 3.3.2) and phenotype-based functions were predicted with BugBase and FAPROTAX [24].

\section{Statistical analyses}

Differences between two groups were determined by one-way analysis of variance. $p$-values of less than 0.05 were considered statistically significant. All statistical tests were carried out using SPSS version 17.0.

\section{Results}

Effect of selenium intake in diet on selenium content of blood and nails

According to the inclusion and exclusion criteria, 50 volunteers from three high-selenium areas and 50 from three low-selenium areas were randomized to participate in the study. The selenium content of their blood and nails were analyzed, showing clear differences (Table 1).

\section{Degree of dietary selenium intake altered the intestinal} microbiota composition

To compare fecal microbial populations of people living in high- and low-selenium area, the $16 \mathrm{~S}$ rRNA phylogenetic approach was applied. We observed 131,389 (84,066-145,017) effective tags in the sequencing. Sequences with at least $97 \%$ similarity were clustered into OTUs and an average of 449 (236-744) OTUs were observed. A Venn diagram (Fig. 1A) displaying the overlapping OTUs for the two groups shows that 1583 of 2834 OTUs accounting for total richness were universal to all the samples, and 746 and 505 OTUs were observed in the high- and lowselenium groups, respectively. The microbial communities were assessed based on phylum, class, order, family, genus, and species between the high- and lowselenium area. Clear diversity was observed, but there was no significant difference between the two control groups (Fig. 1B). At the phylum level, Firmicutes were predominant in the two groups, followed by Bacteroidetes and Proteobacteria, with only a very small proportion of the other phyla. However, the proportion of Bacteroidetes and Proteobacteria was significantly different between the high- and low-selenium area (Fig. 1C). At the phylum level, Bacteroides, Roseburia, Faecalibacterium, Blautia, and Bifidobacterium are observed usually, but no significant difference was shown in the two control groups (Fig. 1D). 
Table 1 Comparison of selenium content in the blood and nails between high- and low-selenium areas

\begin{tabular}{lcccc}
\hline Environment type & Region & Cases & Blood selenium concentration $(\mu \mathrm{g} / \mathrm{mL})$ & Nail selenium concentration $(\mu \mathrm{g} / \mathrm{g})$ \\
\hline High-selenium area & 1 & 13 & $0.129 \pm 0.900$ & $0.837 \pm 0.573$ \\
& 2 & 20 & & $0.362 \pm 0.199$ \\
Low-selenium area & 3 & 17 & $0.057 \pm 0.165$ & \\
\hline
\end{tabular}
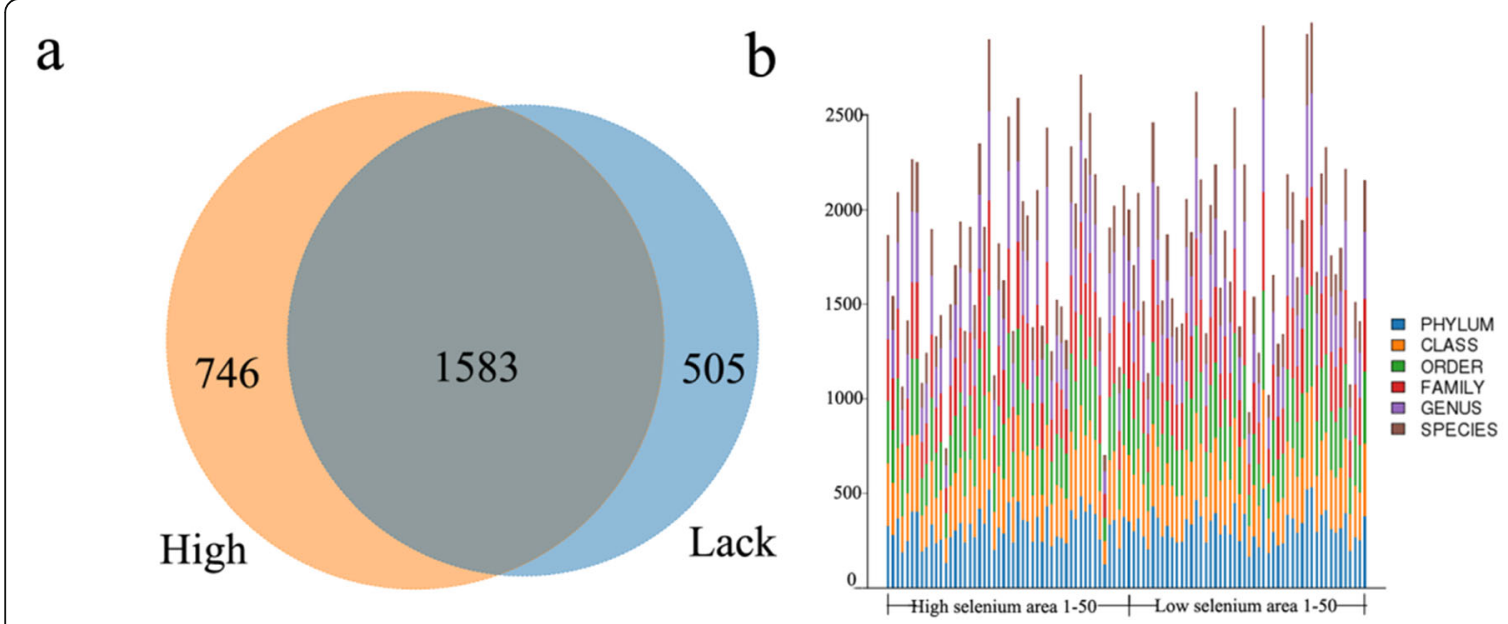

C $100 \%$
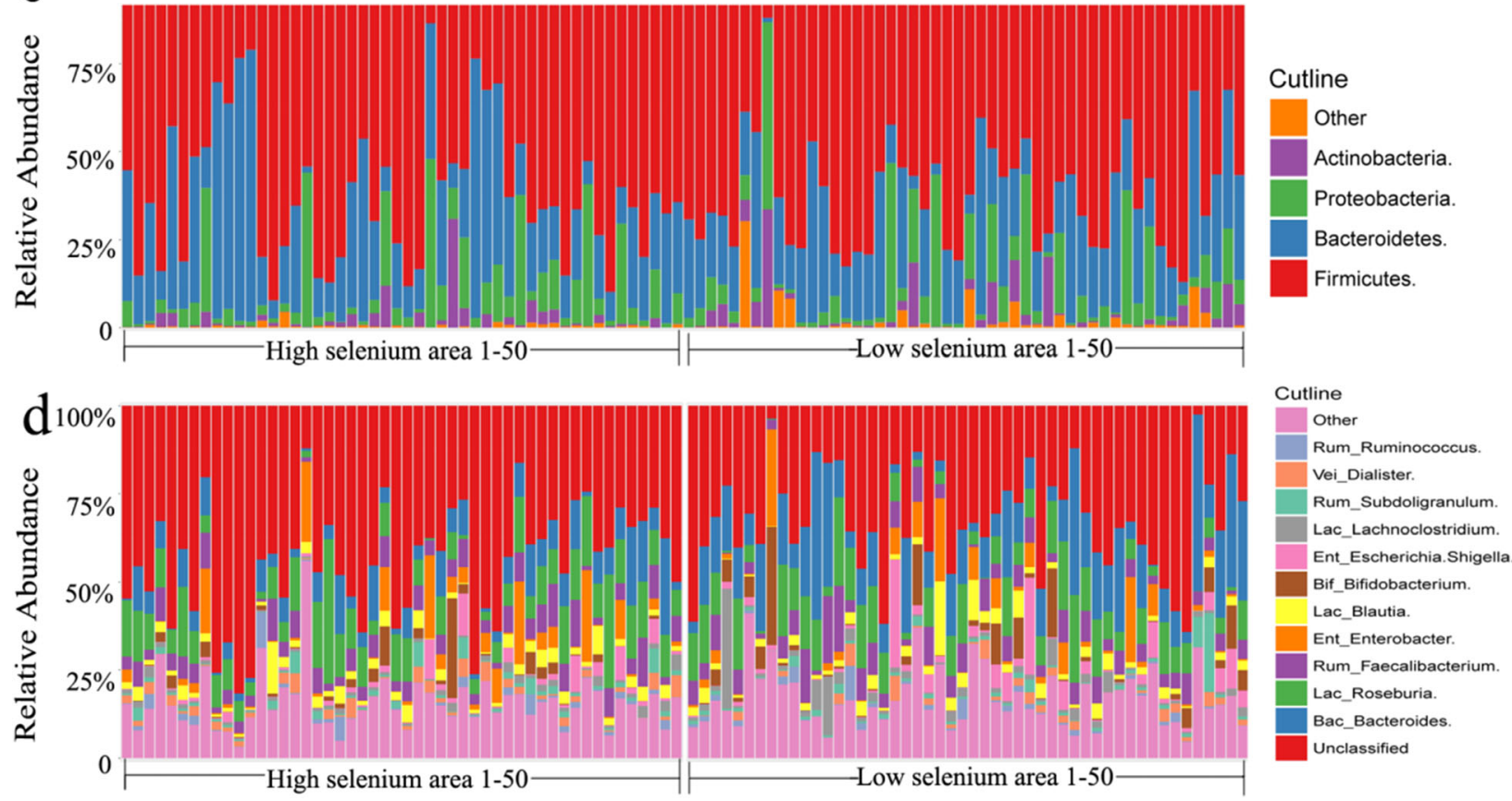

Fig. 1 Difference in dietary selenium intake altered the composition of the intestinal microbiota. Fecal samples were collected from the high- and low-selenium groups and the intestinal microbiota were examined by $16 \mathrm{~S}$ rRNA sequencing. (A) Venn diagram of OTUs showing microbiota differences high- and low-selenium groups, with altered strains altered and overlap. (B) Microbial communities were examined based on phylum, class, order, family, genus, and species in the high- and low-selenium areas. (C) Relative abundance of predominant bacteria at the phylum level. (D) Relative abundance of predominant bacteria at the genus level 


\section{Heatmap analysis of gut microflora in high- and low- selenium areas}

Heatmaps were generated on the basis of the relative abundance of genera and produced by Bray-Curtis dissimilarity (Fig. 2). In total, the 60 most abundant genera of bacteria associated with the 100 volunteers are reflected by the heatmap. Of these, 10 genera were shared by most samples in a higher proportion, including Bactericides, Faecalibacterium, Enterobacter, Dialister, Escherichia-shigella, Roseburia, Bilidobacterium, Alloprevotella, Coprobacter, and Pyramidobacter. Further analysis showed that the relative abundance of genera has greater similarity within each group of volunteers.
Beta diversity analysis of gut microflora in high- and lowselenium areas

Beta diversity analysis was performed to examine the differences between the samples as a method of visualizing similarities or differences in research data (Fig. 3). Each point represents a vector of top several main characteristic values. The red and blue points represent samples in the high- and low-selenium areas, respectively. Principal co-ordinates analysis showed a few features from three dimensions (Fig. 3A1, A2, A3). The orange and blue ellipses represent the main distribution of the high- and lowselenium areas, respectively, and the two groups showed significant differences. Principal component analysis showed dominating features from three dimensions (Fig. 3B1, B2, B3), with slight difference between the two groups. Nonmetric multidimensional scaling compares the differences between the two

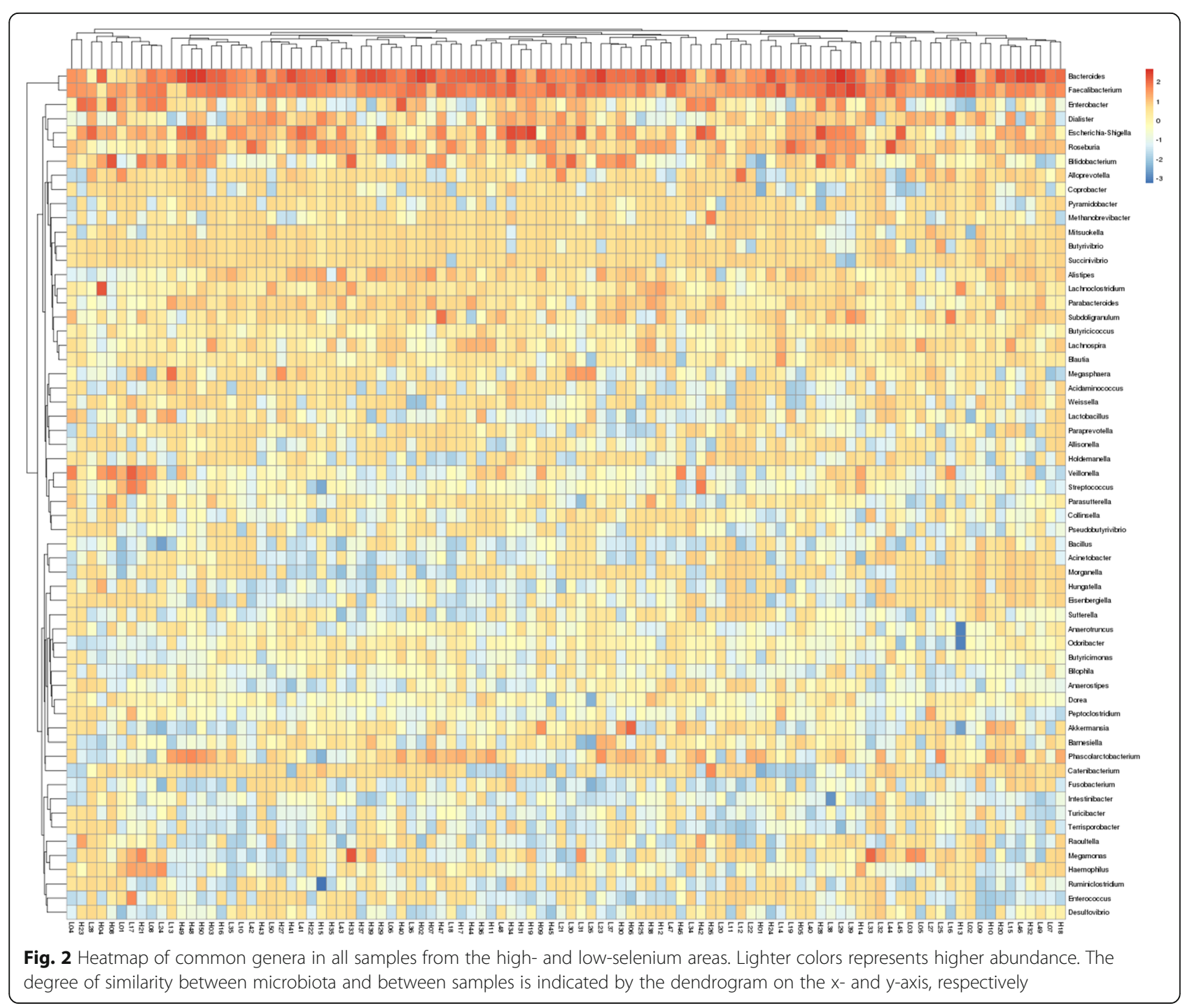



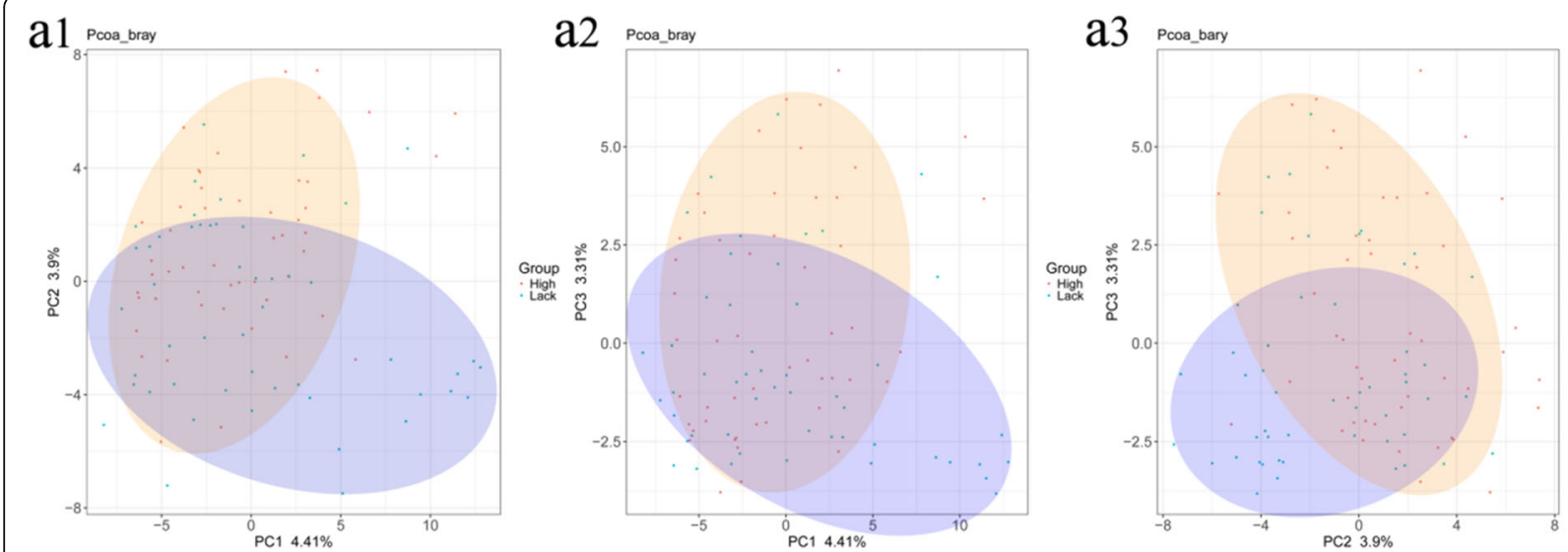

Group
$:$ High
Lack

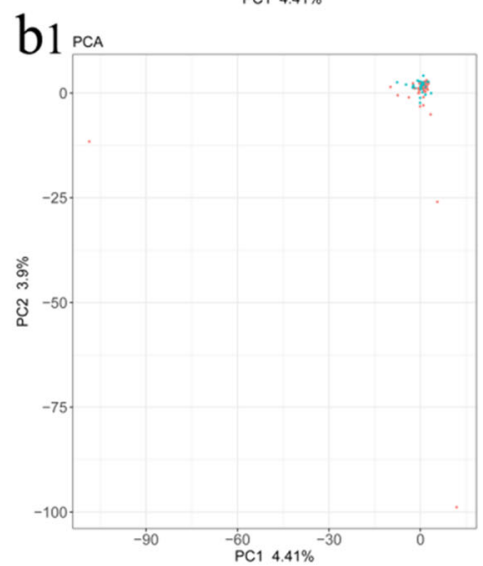

b2
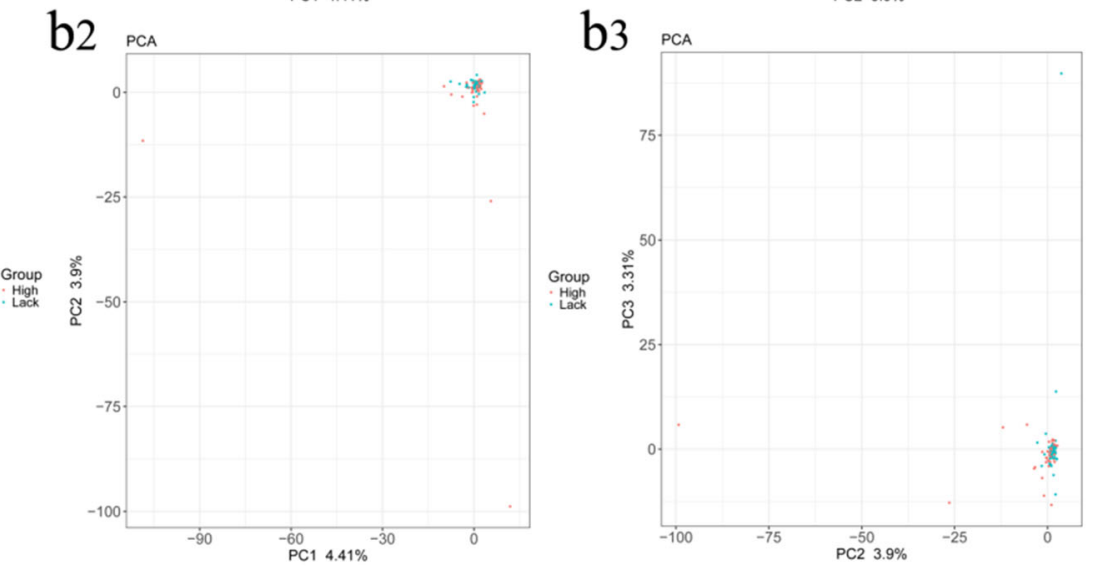

C NMDS

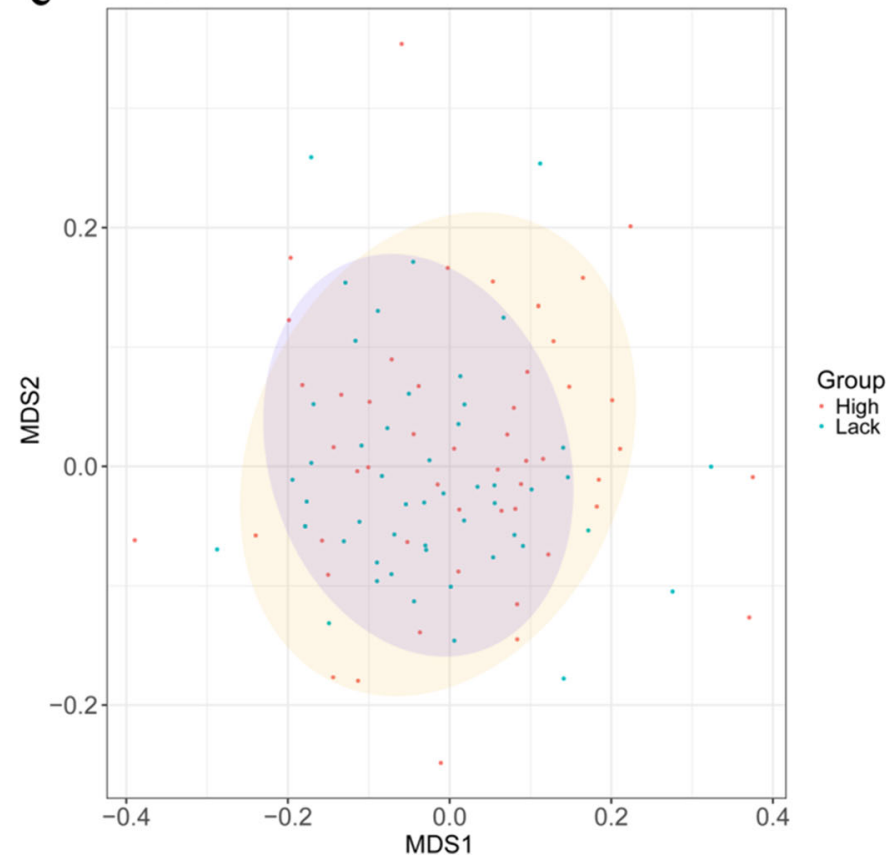

Fig. 3 Beta diversity analysis of gut microflora in high- and low-selenium areas. Orange and blue represent high- and low-selenium area, respectively. $\mathbf{A} 1, \mathbf{A} 2, \mathbf{A} 3$, Principal co-ordinate analysis, a method of visualizing similarities or differences in data. Several features from the beta diversity of gut microflora were observed in three dimensions in high- and low-selenium areas. B1, B2, B3, Principal component analysis. Dominating features from the beta diversity of gut microflora were observed in three dimensions in high- and low-selenium areas. (C) Nonmetric multidimensional scaling comparing the difference between samples based on the number of distance matrices 


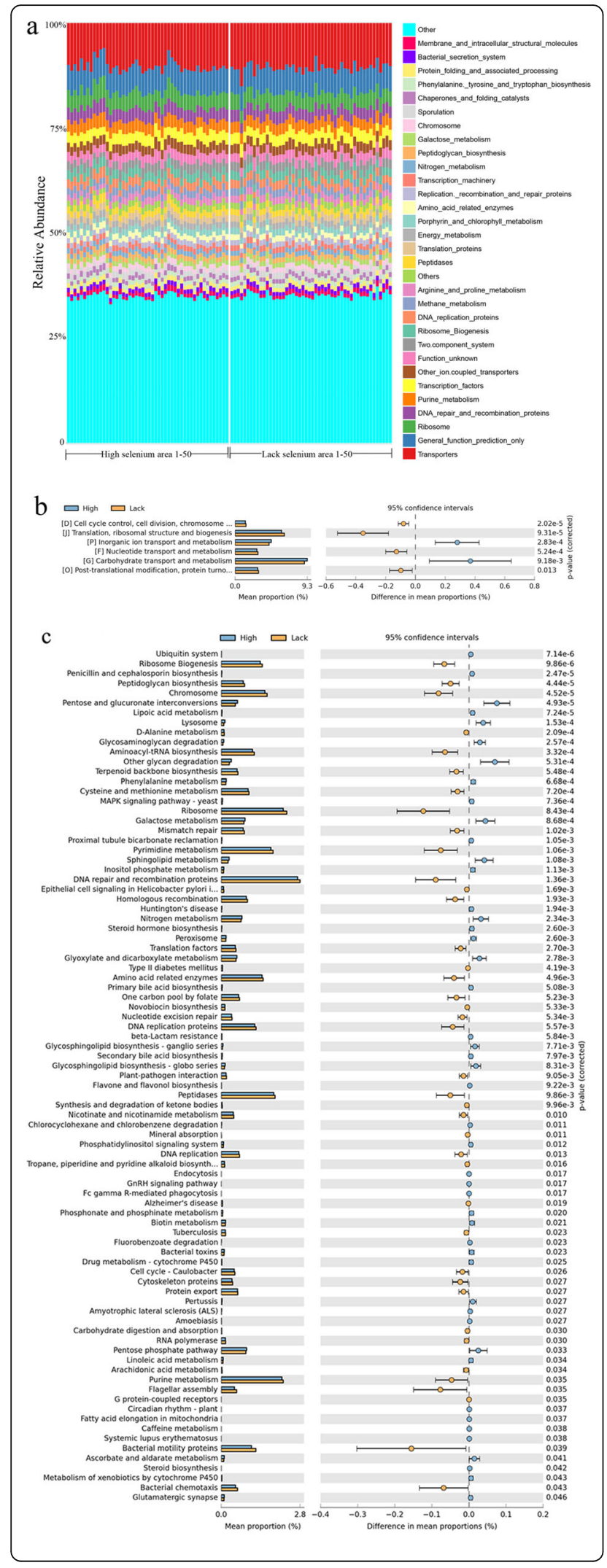

Fig. 4 Variance analysis of gut microflora function in the high- and lowselenium areas. (A) Histogram of top 30 metabolic pathways analyzed by PICRUSt function prediction. Comparison of gut microflora function among 100 volunteers in high- and low-selenium areas. The histogram indicated slight differences in predicted function among individuals, but no significant difference was observed between the two groups. (B) COG analysis diagram indicating significant difference in cell cycle control, inorganic ion transport and metabolism, nucleotide transport and metabolism, carbohydrate transport and metabolism, post-translation modification, and ribosomal structure and biogenesis. (C) Variance analysis of gut microflora function by KEGG pathways in the third layer. Blue and orange represents high- and low-selenium, respectively. Diagram on the left lists the composition with significant differences between groups and proportion of each group, evaluated by KEGG in the third level. Diagram on the right indicates the confidence interval and $p$-value of each group

groups based on the number of distance matrices (Fig. 3B). Data from the high-selenium samples showed more discrete distribution compared to those from the low-selenium samples, with significant differences between them.

\section{PICRUSt, Clusters of Orthologous Groups (COG), and Kyoto Encyclopedia of Genes and Genomes (KEGG)} analysis of gut microflora in high- and low-selenium areas Output data from QIIME were analyzed using PICRUSt enabling the metagenomic make-up of samples to be inferred from the $16 \mathrm{~S}$ data. The top 30 metabolic pathways with significant differences were identified by PICRUSt function prediction. Gut microflora function was compared between 100 volunteers in the high- and low-selenium areas. The histogram indicated a significant difference in predicted function among individuals, but no significant difference between two groups (Fig. 4A). The metabolic profile of bacterial community structure of the 100 volunteers was annotated using COG and KEGG databases. Assembled contigs were analyzed by assigning predicted functions to genes based on COG. In total, 6 classes with significant differences were identified by COG based on functional categories, namely cell cycle control, inorganic ion transport and metabolism, nucleotide transport and metabolism, carbohydrate transport and metabolism, post-translation modification, and ribosomal structure and biogenesis (Fig. 4B). Comparison of fecal samples from high- and low-selenium samples showed significantly different metabolic functions in 87 pathways, as assessed by the level third of KEGG (Fig. 4C), particularly in pathways associated with amino acid synthesis and metabolism, lipoic acid metabolism, ubiquitin system, cysteine and methionine metabolism, DNA repair and recombination proteins, and nitrogen metabolism, glyoxylate and dicarboxylate metabolism and RNA polymerase. 


\section{Phenotype classification of gut microflora in high- and low-selenium areas}

Comparison of eight phenotype classifications was carried out according to the results of the $16 \mathrm{~s}$ high flux and basing on BugBase. No significant difference was observed between the eight phenotype classifications from the high- and low-selenium areas, including grampositive (Fig. 5A), gram-negative (Fig. 5B), stress-tolerant (Fig. 5C), biofilm-forming (Fig. 5D), aerobic (Fig. 5E), anaerobic (Fig. 5F), facultatively anaerobic (Fig. 5 G), and potentially pathogenic (Fig. $5 \mathrm{H}$ ) phenotypes.

\section{Discussion}

Earlier studies have indicated that both high- and lowselenium areas exist, with significant variations in environmental selenium concentration [25]. The concentrations of selenium in the nails of the residents were strongly affected by the environmental selenium content, which was confirmed in this study (Table 1). The concentration of selenium in the blood and nail in the highselenium area is significantly higher than that in the low-selenium area. In addition, residents in Enshi have similar genetic origins, living conditions, eating habits, and climate, providing a unique environment to study the correlation between selenium and human health. Thus, we focused on studying the relationship between selenium uptake and human gut microbiota base on this geographic feature.
In this study, there were several main reasons that elderly people were chosen as the subjects. In addition to obvious differences in dietary selenium intake, the living conditions of the subjects are relatively fixed, their life habits are similar, and they consume a basic diet. At the same time, there is a distinct association between age and gut microbiota composition [26]. The selenium content in the blood and nails confirmed that high- and low-selenium areas exhibited obvious differences. The intestinal microbiota is affected by many factors, such as environment, diet, and age. In this study, we showed that the dietary intake of the trace element selenium undoubtedly also has a significant impact on the intestinal microbiota. Our findings provide a valuable reference that can offer new clues and ideas for the study of selenium in human health.

We also observed distinct taxonomic associations of selenium in the gut microbiota, which were most apparent at the OTU level where a number of significant associations were observed between the two groups. Differences in dietary selenium intake altered the composition of the intestinal microbiota (Fig. 1). Significant differences in the number and distribution of OTUs were observed between the two groups, but it is also reflected in two aspects. One is the various bacteria distributed in phylum, class, order, family, genus, and species, and the other is the relative abundance of predominant bacteria at the phylum and genus levels. Heatmap analysis of common genera, beta diversity analysis, and phenotype classification based on BugBase
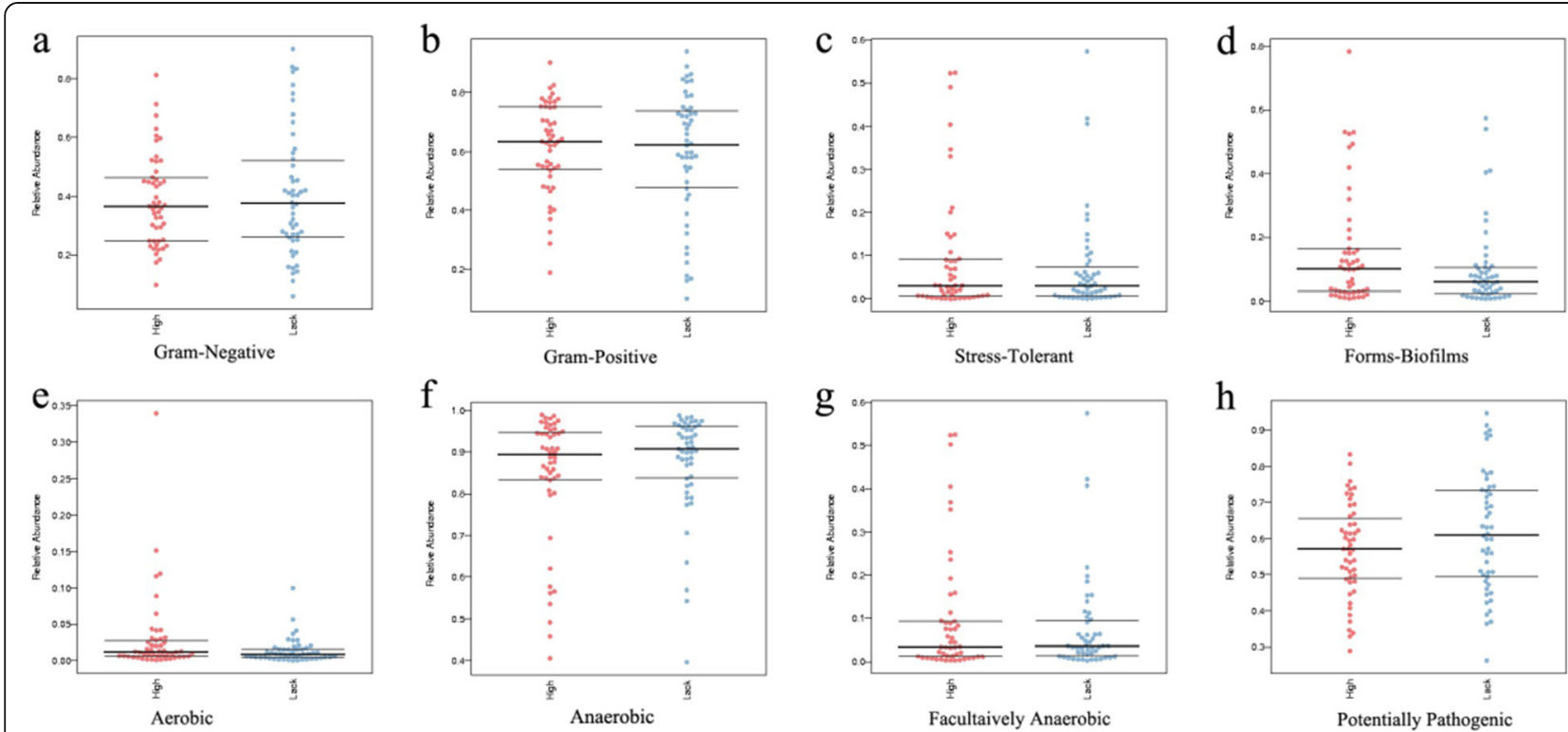

Fig. 5 Comparison of eight phenotype classifications based on the result of $16 \mathrm{~S}$ high flux and based on BugBase. (A) Gram-positive phenotype. (B) Gram-negative phenotype. (C) Stress-tolerant phenotype. (D) Biofilm-forming phenotype. (E) Aerobic phenotype. (F) Anaerobic phenotype. (G) Facultatively anaerobic phenotype. (H). Potentially pathogenic phenotype 
showed a clear difference in gut microbiota between high- and low-selenium areas. Associating the above analyses with OTUs, the comprehensive results revealed that the difference in selenium intake is one of the main influencing factors that impact the intestinal flora. Further studies will be required to investigate the importance of specific taxa associated with selenium intake. This study has some limitations as the gut microbiota composition was only analyzed using $16 \mathrm{~S}$ rRNA amplicon analysis. The use of whole-metagenome sequencing would provide more accurate species level assignments and direct functional information [27].

This study focused on function prediction and metabolism pathways with PICRUSt, COG, and KEGG based on OTUs. Significant differences were identified in a number of metabolism pathways, indicating their association with selenium intake. Particularly, pathways involved in synthesis and degradation of ketone bodies, which were closely associated with cystic fibrosis [28], were observed. Metabolism pathways involved in type II diabetes were also observed, and its correlation with selenium has attracted the attention of medical researchers. Park et al. reported the correlation between higher toenail selenium content with lower risk of type II diabetes [29] and Stranges et al. studied the effects of long-term selenium supplementation on the incidence of type II diabetes [30]. It is reasonable to deduce that the gut microbiota plays an important role in the effect of selenium in type II diabetes. Metabolism pathways involved in Alzheimer's disease (AD) is our focus because it is a current research highlight. Cardoso et al. [31] and Vural et al. [32] demonstrated that AD patients had lower selenium levels than healthy elderly people, and the effect of the gut microbiota on cognitive behavioral capability was demonstrated to play a critical role in the pathogenesis of $\mathrm{AD}$ through the microbiota-gut-brain axis [33]. It is clear that there is a close link between selenium, the gut microbiota, and $\mathrm{AD}$, and this will be explored in future studies.

\section{Conclusion}

In this study, we took advantage of the different distributions of selenium in the Enshi region and studied the effect of dietary selenium intake on the intestinal microflora. We showed that differences in the selenium intake in the elderly population significantly impacted the function and metabolism of gut microbes and may be associated with certain diseases. This study provides new ideas and directions for studying the correlation between selenium and human health.

\section{Abbreviations}

OUT(s): Operational Taxonomic Unit(s); PCoA: Principal co-ordinates analysis; PCA: Principal component analysis; NMDS: Nonmetric Multidimensional Scaling; AD: alzheimer's disease; COG: Clusters of Orthologous Groups of proteins; KEGG: Kyoto Encyclopedia of Genes and Genomes; STAMP: Statistical Analysis of Metagenomic Profiles

\section{Acknowledgements}

Not applicable.

\section{Authors' contributions}

Zi-xiong Zhang: experiment design, quality control, article modification. Hua Xiang: experiment performance. Guo-gen Sun: wrinting article. Yan-hua Yang: $16 \mathrm{~S}$ sequencing data analysis. Chen Chen: director of experimental design. Tuo Li: supporting fund of project and providing advice of research. The author(s) read and approved the final manuscript.

\section{The number of ethical approval}

2021-005-01.

\section{Funding}

Enshi State Science and Technology Program Project (XYJ2016000306).

Availability of data and materials

Not applicable.

\section{Declarations}

\section{Ethics approval and consent to participate}

The experimental protocol was established, according to the ethical guidelines of the Helsinki Declaration and was approved by the Human Ethics Committee of Ethics Committee of Central Hospital of Enshi Tujia and Miao Autonomous Prefecture. Written informed consent was obtained from individual or guardian participants.

\section{Consent for publication}

Not applicable.

\section{Competing interests}

The authors declare that they have no competing interests.

\section{Author details}

${ }^{1}$ Institute of Selenium and Human Health of Hubei, Hubei Province, Enshi City, China. ${ }^{2}$ Central Hospital of Enshi Autonomous Prefecture, Enshi Autonomous Prefecture, Hubei Province, Enshi City, China. ${ }^{3}$ Research Institute of Otolaryngology Head and Neck Surgery, Renmin Hospital of Wuhan University, Wuhan, China.

Received: 16 March 2021 Accepted: 12 August 2021

Published online: 13 December 2021

\section{References}

1. Helander H.F., Fandriks L. Surface area of the digestive tract - revisited. Scand J Gastroenterol. 2014;49(6):681-689. doi: https://doi.org/10.3109/003 65521.2014 .898326$.

2. Hooper LV, Wong MH, Thelin A, Hansson L, Falk PG. Gordon J.I. Molecular analysis of commensal host-microbial relationships in the intestine. Science. 2001;291:881-4.

3. Eckburg PB, Bik EM, Bernstein CN, Purdom E, Dethlefsen L, Sargent M. Diversity of the human intestinal microbial flora. Science. 2005;308:1635-8.

4. Nam YD, Jung MJ, Roh SW, Kim MS, Bae JW. Comparative analysis of Korean human gut microbiota by barcoded pyrosequencing. PLoS One. 2011;6(7): e22109.

5. Tilg H, Kaser A. Gut microbiome, obesity, and metabolic dysfunction. J Clin Invest. 2011;121:2126-32.

6. Xu XF, Xu PP, Ma CW. Gut microbiota, host health, and polysaccharides. Biotechnol Adv. 2013;31:318-37.

7. Li M, Wang B, Zhang M, Rantalainen M, Wang S, Zhou H. Symbiotic gut microbes modulate human metabolic phenotypes. Proc Natl Acad Sci USA. 2008;105:2117-22. Lee YK, Mazmanian SK. Has the microbiota played a critical role in the evolution of the adaptive immune system. Science. 2010; 330:1768-73.

8. Lee YK, Menezes JS, Umesaki Y, Mazmanian S. Proinflaamatory T-cell responses to gut microbiota promote experimental autoimmune encephalomyelitis. Proc Natl Acad Sci USA. 2011;108(Suppl. 1):4615-22. 
9. Brown CT, Davis-Richardson AG, Giongo A, Gano KA, Crabb DB, Mukherjee $\mathrm{N}$. Gut microbiome metagenomics analysis suggests a functional model for the development of autoimmunity for type 1 diabetes. PLoS One. 2011; 6(10):e25782.

10. Kerckhoffs AP, Ben-Amor K, Samsom M, van der Rest ME, de Vogel J, Knol J. Molecular analysis of faecal and duodenal samples reveals significantly higher prevalence and numbers of Pseudomonas aeruginosa in irritable bowel syndrome. J Med Microbiol. 2011;60:236-45.

11. Zhu Y, Michelle LT, Jobin C, Young HA. Gut microbiota and probiotics in colon tumorigenesis. Cancer Lett. 2011;309:119-27.

12. Asquith $M$, Powrie F. An innately dangerous balancing act: intestinal homeostasis, inflammation, and colitis-associated cancer. J Exp Med. 2010; 207:1573-7.

13. Turnbaugh PJ, Hamady M, Yatsunenko T, Cantarel BL, Duncan A. Ley R.E. A core gut microbiome in obese and lean twins. Nature. 2009a;457:480-4.

14. Arumugam M, Raes J, Pelletier E, Le Paslier D, Yamada T. Mende D.R. Enterotypes of the human gut microbiome. Nature. 2011;473:174-80.

15. Fahey GC Jr, Barry KA, Swanson KS. Age-related changes in nutrient utilization by companion animals. Annu Rev Nutr. 2008;28:425-45.

16. Sharon G, Segala D, Ringob JM, Hefetzc A, Zilber-Rosenbergd I, Rosenberga E. Commensal bacteria play a role in mating preference of Drosophila melanogaster. Prod Natl Acad Sci USA. 2010;107:20051-6.

17. De Filippo C, Cavalieria D, Di Paolab M, Ramazzottic M, Poulletd JB. Massartd S.Impact of diet in shaping gut microbiota revealed by a comparative study in children from Europe and rural Africa. Proc Natl Acad Sci USA. 2010;107:14691-7.

18. Daniela P., Mary A., Uyoga, Michael B., Zimmermann. Iron Fortification of Foods for Infants and Children in Low-Income Countries: Effects on the Gut Microbiome, Gut Inflammation, and Diarrhea. Nutrients. 2016 ; 8(8): 494.

19. Feng CX, Liu JJ, Liu S, Hu RZ, Chi GX. Petrogenesis and sedimentary environment of the cherts from Yutangba, western Hubei Province: Evidence from silicon, oxygen, carbon and sulfur isotopic compositions. Acta Petrologica Sinica. 2009;25(5):1253-9.

20. Kieliszek M, Błażejak S. Selenium: significance, and outlook for supplementation. Nutrition. 2013;29(5):713-8.

21. Zhu JM, Wang N, Li SH, Li L, Su HC. Distribution and transport of selenium in Yutangba, China: Impact of human activities. Sci Total Environ. 2008;392: 252-61.

22. Caporaso JG, Kuczynski J, Stombaugh J, Bittinger K, Bushman FD, Costello EK, Fierer N, Peña AG, Goodrich J. K. QIIME allows analysis of highthroughput community sequencing data. Nat Methods. 2010;7(5):335-6. doi: https://doi.org/10.1038/nmeth.f.303

23. Segata N, Izard J, Waldron L, Gevers D, Miropolsky L, Garrett WS, Huttenhower C. Metagenomic biomarker discovery and explanation. Genome Biol. 2011;12(6):R60. doi:https://doi.org/10.1186/gb-2011-12-6-r60.

24. Parks DH, Tyson GW, Hugenholtz P, Beiko RG STAMP: Statistical analysis of taxonomic and functional profiles. Bioinformatics. 2014; 30:3123-3124.

25. Langille MGl, Zaneveld J, Caporaso JG, McDonald D, Knights D, Reyes JA Predictive functional profiling of microbial communities using 165 rRNA marker gene sequences. Nat Biotech. 2013;31:814-21.

26. Zhu JM, Zheng BS. Distribution of Se in mini-landscape of Yutangba, Enshi, Hubei Province China. Appl Geochem. 2001;16:1333-44.

27. Jackson MA, Jackson M, Jeffery IB, Beaumont $M$, Bell JT, Clark AG, Ley RE, OToole PW, Spector TD. Steves C.J. Signatures of early frailty in the gut microbiota. Genome Med. 2016:8:8. doi:https://doi.org/10.1186/s13073-0160262-7.

28. Gill SR, Pop M, DeBoy RT, Eckburg PB, Turnbaugh PJ. Samuel B.S. Metagenomic analysis of the human distal Gut microbiome. Science. 2006; 312:1355-9. doi:https://doi.org/10.1126/science.1124234.

29. Pamela V., Federica D.C., Andrea Q. A. Metagenomic and in Silico Functional Prediction of Gut Microbiota Profiles May Concur in Discovering New Cystic Fibrosis Patient-Targeted Probiotics. Nutrients. 2017 Dec; 9(12): 1342.

30. Park K, Rimm EB, Siscovick DS, Spiegelman D, Manson JE, Morris JS, Hu FB, Mozaffarian D. Toenail selenium and incidence of type 2 diabetes in U.S. men and women. Diabetes Care. 2012;35:1544-51. doi:https://doi.org/10.233 7/dc11-2136.

31. Stranges S, Marshall JR, Natarajan R, Donahue RP, Trevisan M, Combs GF Cappuccio FP, Ceriello A, Reid ME. Effects of Long-Term Selenium Supplementation on the Incidence of Type 2 Diabetes: A Randomized Trial. Ann Intern Med. 2007;147:217-23. doi:https://doi.org/10.7326/0003-4819-14 7-4-200708210-00175.
32. Cardoso BR, Ong TP, Jacob-Filho W, Jaluul O, Freitas MI, Cozzolino SM. Nutritional status of selenium in Alzheimer's disease patients. Br J Nutr. 2010;103(6):803-6.

33. Vural H, Demirin H, Kara Y, Eren I, Delibas N. Alterations of plasma magnesium, copper, zinc, iron and selenium concentrations and some related erythrocyte antioxidant enzyme activities in patients with Alzheimer's disease. J Trace Elem Med Biol. 2010;24(3):169-73.

34. Zhu X.Q., Han Y., Du J., Liu R.Z. Microbiota-gut-brain axis and the central nervous system. Oncotarget. 2017 Aug 8; 8(32): 53829-53838.

\section{Publisher's Note}

Springer Nature remains neutral with regard to jurisdictional claims in published maps and institutional affiliations.
Ready to submit your research? Choose BMC and benefit from:

- fast, convenient online submission

- thorough peer review by experienced researchers in your field

- rapid publication on acceptance

- support for research data, including large and complex data types

- gold Open Access which fosters wider collaboration and increased citations

- maximum visibility for your research: over $100 \mathrm{M}$ website views per year

At BMC, research is always in progress.

Learn more biomedcentral.com/submissions 